\title{
CHARACTERIZATIONS OF ALGEBRAS ARISING FROM LOCALLY COMPACT GROUPS
}

\author{
PAUL L. PATTERSON
}

\begin{abstract}
Two Banach *-algebras are naturally associated with a locally compact group, $G$ : the group algebra, $L^{1}(G)$, and the measure algebra, $M(G)$. Either of these Banach algebras is a complete set of invariants for $G$.

In any Banach *-algebra, $A$, the norm one unitary elements form a group, $S$. Using $S$ we characterize those Banach *-algebras, $A$, which are isometrically ${ }^{*}$-isomorphic to $M(G)$. Our characterization assumes that $A$ is the dual of some Banach space and that its operations are continuous in the resulting weak * topology. The other most important condition is that the convex hull of $S$ must be weak* dense in the unit ball of $A$.

We characterize Banach *-algebras which are isomerically isomorphic to $L^{1}(G)$ for some $G$ as those algebras, $A$, whose double centralizer algebra, $D(A)$, satisfies our characterization for $M(G)$. In addition we require $A$ to consist of those elements of $D(A)$ on which $S$ (defined relative to $D(A)$ ) acts continuously with its weak* topology. Using another characterization of $L^{1}(G)$ we explicitly calculate the above isomorphism between $A$ and $L^{1}(G)$.
\end{abstract}

\section{INTRODUCTION}

Group algebras and operator algebras are the most important examples of noncommutative Banach *-algebras. Several characterizations of operator algebras have proven important. Norm closed *-algebras of operators on a Hilbert space were characterized by M. A. Naimark and I. M. Gelfand in 1943 [5] (cf., R. S. Doran and Victor A. Belfi, Characterizations of $C^{*}$-algebras, Marcel Dekker, New York, 1986, for further developements of this characterization). Weakly closed *-algebras of operators were characterized in 1956 [17]. Both of these characterizations have played important roles in the theory. Thus it is surprising that characterizations of group algebras as Banach *-algebras have not been given. This paper is devoted to such characterizations for the two most important examples of group algebras of locally compact groups.

In $1952 \mathrm{~J}$. G. Wendel showed that the algebra $L^{1}(G)$, of all absolutely integrable functions on $G$ considered as a Banach algebra (with convolution multiplication), characterizes the locally compact group $G$ up to homeomorphic isomorphism [20]. In 1964 B. Johnson using Wendel's result proved that $M(G)$,

Received by the editors March 26, 1990.

1991 Mathematics Subject Classification. Primary 43A10, 43A20; Secondary 43A22.

This paper formed a portion of the author's Ph.D. thesis at the University of Oregon, (August, 1987). The author is grateful to Professor Theodore W. Palmer for his direction and assistance. The author would also like to thank the referee for his suggestions concerning Theorem 4.9. 
the algebra of complex regular Borel measures on $G$, (with convolution multiplication and the total variation norm), is also a complete set of invariants for $G[10]$.

Both of these group algebras have natural involutions but the involutions where not used by either Wendel or Johnson. Their results raise the interesting question of the extent to which the involution of these two group algebras are determined by their structure as Banach algebras. In a subsequent paper, using methods of the present paper, we will determine all isometric involutions on $L^{1}(G)$ and $M(G)$ and show which ones arise from the group algebra structure.

Although no work has been done on characterizing group algebras as Banach *-algebras there has been some work done on characterizing them as Banach algebras. Results of this paper are related to R. Rigelhof's 1969 characterization of $M(G)$ as a Banach algebra for $G$ a arbitrary locally compact group [15]. His characterization uses properties of the set, $E$, of extreme points of the unit ball and does not involve the involution. For $E$ we subsitute the closely related set $S$ of norm one unitaries. The addition structure provided by the involution simplifies some of his assumptions. In particular it removes the need for assumption (4) of Theorem 1 , since $S$ is obviously a group. In an appendix to this paper we discuss an obscurity in the proof given in [15].

Somewhat more satisfying characterizations of $L^{1}(G)$ and $M(G)$ as Banach algebras have been found in special cases. In $1965 \mathrm{M}$. Rieffel characterized both $L^{1}(G)$ and $M(G)$ for abelian groups $G$ [14]. His main hypothesis is the existence of a sufficient number of multiplicative functionals, which induce a complex $L$-space order on the Banach algebra, to separate points. Thus his technique will not work for nonabelian groups.

In $1966 \mathrm{~F}$. Greenleaf characterized $L^{1}(G)$ for compact groups $G$ [6]. $\mathrm{He}$ studied the left centralizers of $L^{1}(G)$, i.e. the linear functions $T: L^{1}(G) \mapsto$ $L^{1}(G)$ satisfying $T(f * g)=T(f) * g$. His main axiom is that left multiplication by any element of norm one should be the strong operator limit of convex sums of left centralizers. This axiom characterizes a slightly larger class of Banach algebras than $L^{1}(G)$ for $G$ a compact group. He then characterizes those algebras that are $L^{1}(G)$ by the use of a second axiom that involves the minimal left ideals of the algebra. Since $L^{1}(G)$ for noncompact groups has no minimal one-sided ideals, Greenleaf's method will not work for the noncompact case.

It is easier to characterize $M(G)$ than $L^{1}(G)$ since $G$ may be regarded as the subset of $M(G)$ consisting of the point masses. The set of unitaries of norm one in $M(G)$, which we denote by $S$, is just the set of multiplies of point masses by complex numbers of norm one. Thus, with the weak* topology, (obtained by considering $M(G)$ as the dual of the Banach space of continuous functions which vanish at infinity), $S$ can be identified as a topological group with $\mathbf{T} \times G$.

In characterizing which unital Banach*-algebras $A$ are isometrically *-isomorphic to $M(G)$ we assume the existence of a Banach space, $V$, such that $V^{*}=A$. Let $S$ be the set of unitaries in $A$ of norm one. In order to separate the factors in $\mathbf{T} \times G$, we must assume that $A$ has a multiplicative linear functional which can play the role of $\mu \mapsto \mu(g)$. We then assume that $A$ satisfies conditions that show $S$ with the weak ${ }^{*}$ topology induced by $V$ is a topological group. To insure that $S$ is locally compact we make the slightly 
stronger assumption that $S \cup\{0\}$ is weak * closed. We are then able to define a homomorphism from $M(G)$ to $A$. The assumption that the convex hull of $S$ is weak* dense in the unit ball of $A$ guarantees that our map is onto. Besides showing which unital Banach *-algebras are isometrically *-isomorphic to $M(G)$ we also characterize $M(G) / N$ for $N$ a weak * closed *-ideal of $M(G)$.

The task for $L^{1}(G)$ is more difficult since $G$ is not embedded in $L^{1}(G)$. However the double centralizer algebra, $D\left(L^{1}(G)\right)$, of $L^{1}(G)$ is $M(G)$. Hence we may use our characterization of $M(G)$. We use the strict topology induced by the two-sided ideal $L^{1}(G)$ on $M(G)$.

The paper is organized as follows. In $\S 1$ we give the necessary definitions, facts and notation from functional analysis, locally compact group theory, and measure theory. The characterization of $M(G)$ and related results are given in $\S 2$. Section 3 contains a brief review of those facts about the double centralizer algebra and the strict topology needed in the characterization of $L^{1}(G)$. Two characterizations of $L^{1}(G)$ are given in $\S 4$. The first uses our characterization of $M(G)$ from $\S 2$. The second contains strong enough assumptions to allow an explicitly calculation of the isomorphism obtained in our first characterization of $L^{1}(G)$ and to show that it is similar to the map used by Greenleaf in [6].

\section{Preliminaries}

Denote by $\mathbf{R}, \mathbf{C}$, and $\mathbf{T}$ the real and the complex numbers and the one torus $\mathbf{T}=\{\xi \in \mathbf{C}:|\xi|=1\}$ respectively. We assume the reader is familar with basic topological group theory, measure theory, and functional analysis (cf., [8], [9] for topological groups and measure theory and [16] for functional analysis).

Functional Analysis. Let $A$ be a Banach space. Denote its dual, the Banach space of norm continuous linear functionals, by $A^{*}$, and let $A_{1}=\{a \in A:\|a\|$ $\leq 1\}$. We use $\kappa$ to denote the natural injection of $A$ into its double dual, $A^{*}$. If $V$ is a subset of $A^{*}$, let the $\sigma(A, V)$-topology be the weakest topology on $A$ in which all the elements of $V$ are continuous. We refer to the $\sigma\left(A^{*}, \kappa(A)\right)$-topology as the weak * topology.

A subspace, $V$, of $A^{*}$ is said to be a minimal subspace if $\gamma: A \mapsto V^{*}$ given by, $\gamma(a)(\omega)=\omega(a)$ for $a \in A$ and $\omega \in V$, is an isometric isomorphism onto $V^{*}$, where $V^{*}$ is the norm continuous linear functionals on $V$. In [2] Diximier gives several equivalent definitions of a minimal subspace. We will make extensive use of one of them, namely $V$ is a norm closed subspace of $A^{*}$ and $V$ is weak* dense in $A^{*}$. Thus if $A$ is a Banach space then $\kappa(A)$ is a minimal subspace of $A^{*}$.

A conjugate linear involutive map from a Banach space to itself will be called a linear involution. If $A$ has a continuous linear involution define an induced linear involution on $A^{*}$ by $\omega^{*}(a)=\omega\left(a^{*}\right)^{-}$for $\omega \in A^{*}$ and $a \in A$. If this involution of $A$ is induced to $A^{*}$ and then the induced involution on $A^{*}$ is induced to $A^{* *}, \kappa$ satisfies $\kappa\left(a^{*}\right)=\kappa(a)^{*}$. If $A$ is a unital *-algebra let $A_{U}$ equal $\left\{a \in A: a^{*} a=1\right\}$.

Groups. By a locally compact group we mean a locally compact Hausdorff group.

Let $G$ be locally compact group. Denote the space of all continuous complex valued functions on $G$ which vanish at infinity by $C_{0}(G)$. Then $C_{0}(G)$ is a Banach algebra with pointwise addition and multiplication and the supremum 
norm, $\|\cdot\|_{\infty}$. We define an isometric involution on $C_{0}(G)$ by $f^{-}(u)=f(u)^{-}$, for all $u \in G$.

Let $M(G)$ denote the Banach space of complex Borel measures on $G$, where the norm is the total variation norm. With the convolution product $M(G)$ is a Banach algebra. It is well known that the pairing,

$$
\langle f, \mu\rangle=\int_{G} f(v) d \mu(v), \quad f \in C_{0}(G), \mu \in M(G),
$$

gives an isometric isomorphism of $M(G)$ onto $C_{0}(G)^{*}[8,14.10]$. For $u \in G$ let $\delta_{u}$ be the element defined by, $\left\langle f, \delta_{u}\right\rangle=f(u)$. Then $\delta_{e}$ is a multiplicative identity for $M(G)$. Using the above pairing and the involution on $C_{0}(G)$ we define the induced involution on $M(G)$ :

$$
\left\langle f, \mu^{*}\right\rangle=\left\langle f^{*}, \mu\right\rangle^{-}, \quad f \in C_{0}(G), \mu \in M(G) .
$$

This is an isometric involution on $M(G)$. With this involution $M(G)$ is a unital Banach *-algebra. The previous section implies that $\kappa\left(C_{0}(G)\right) \subset M(G)^{*}$ is a minimal subspace, and $M(G)^{*}$ is invariant under the induced involution from $M(G)$.

For a locally compact group, $G$, let $\lambda$ and $\Delta$ denote left Haar measure and the modular function on $G$ respectively. Let $L^{1}(G)$ denote the Banach space of all absolutely integrable (with respect to Haar measure) complex valued functions. It is well known that $L^{1}(G)$ is isometerically *-isomorphic to the closed *-ideal in $M(G)$ of the measures which are absolutely continuous with respect to Haar measure cf., $[8,19.18]$. The restriction of the convolution and the involution on $M(G)$ to $L^{1}(G)$ is given by

$$
f * g(v)=\int_{G} f\left(v u^{-1}\right) g(u) d \lambda(u), \quad f, g \in L^{1}(G),
$$

and

$$
\begin{gathered}
f^{*}(v)=\Delta\left(u^{-1}\right) f\left(u^{-1}\right)^{-}, \quad f \in L^{1}(G) . \\
\text { 2. A CHARACTERIZATION OF } M(G) \\
\text { IN TERMS OF ITS UNITARY ELEMENTS }
\end{gathered}
$$

In Theorem 2.3 we will show that for a locally compact group $G, M(G)$ satisfies the conditions of the following theorem with $V=C_{0}(G)$ and $\omega(\mu)=$ $\int_{G} d \mu$.

Theorem 2.1. Let $A$ be a unital Banach *-algebra with isometric involution satisfying the following six conditions:

(1) There is a minimal subspace $V \subset A^{*}$ such that,

(2) $V$ is invariant under the involution induced on $A^{*}$.

(3) If $S=A_{U} \cap A_{1}$ then $S \cup\{0\}$ is $\sigma(A, V)$-closed.

(4) Multiplication in $A$ is separately $\sigma(A, V)$-continuous.

(5) The $\sigma(A, V)$-closure of $\operatorname{co}[S]$ is equal to $A_{1}$.

(6) There exists a nonzero multiplicative linear functional (hence norm continuous see $[16, p .231]), \omega$, which is $\sigma(A, V)$-continuous on $S$.

Then there exists a locally compact group $G$ and a $\sigma\left(M(G), C_{0}(G)\right)$-closed *-ideal $N$ of $M(G)$ with $A$ isometrically *-isomorphic to $M(G) / N$.

Proof. Let $G$ equal $\{s \in S: \omega(s)=1\}$. Clearly $(S, \cdot)$ is a group with $s^{-1}=s^{*}$ and with identity 1. Since for $s \in S, \omega(s)=1=\omega\left(s^{*} s\right)=\omega\left(s^{*}\right) \cdot \omega(s)$, 
one sees that $G$ is a subgroup of $S$. A straightfoward calculation shows that $(\lambda, s) \mapsto \lambda \cdot s$ is a group isomorphism of $\mathbf{T} \times G$ onto $S$. Give both $S$ and $G$ the relative $\sigma(A, V)$-topology.

Condition (2) above implies that $(*)$ is a $\sigma(A, V)$-continuous involution on $A$. The $\sigma(A, V)$-continuity of $(*)$ together with the $\sigma(A, V)$-continuity of $\omega$ on $S$ imply that the inverse operation on $S$ is continuous. Condition (4) above and the $\sigma(A, V)$-continuity of $\omega$ imply the group operation on $G$ is separately continuous. More is true.

Claim 1. $G$ with the relative $\sigma(A, V)$-topology is a locally compact group.

Proof. Define $m: \mathbf{T} \times G \mapsto S$ by $m(\lambda, s)=\lambda s$. It is easy to see that $m$ is oneto-one, onto and continuous. Condition (6) implies that $m^{-1}$ is continuous so $m$ is a homeomorphism. Also $S \cup\{0\}$ is $\sigma(A, V)$-compact, since $S \cup\{0\}$ is a $\sigma(A, V)$-closed subset of a $\sigma(A, V)$-compact set $A_{1}$. Thus $S$ is $\sigma(A, V)$ locally compact. Since $\mathbf{T} \times G$ is homeomorphic to $S$, Lemma A.1 implies that $G$ is a locally compact topological space. Finally $G$ has a separately continuous group operation thus by a theorem of Ellis [4], $G$ is a locally compact group.

Define $\theta: V \mapsto C(G)$ by $\theta(f)(s)=f(s)$, for all $f \in V$ and $s \in G$. Since $\theta$ is simply the restriction of the function $f \in A^{*}$ to $G$, and $S \cup\{0\}$ is $\sigma(A, V)$-compact the next claim is immediate.

Claim 2. If $f \in V$ then $\theta(f)$ is an element of $C_{0}(G)$.

Since $V$ is a minimal subspace of $A^{*}$, there exists $\chi: V^{*} \mapsto A$ with $\chi$ an isometric isomorphism. Using the usual pairing, $\langle$,$\rangle , we have the following$ diagram of maps:

$$
A \stackrel{\chi}{\stackrel{\chi}{\longleftarrow}} \begin{array}{lll}
V^{*} & \stackrel{\theta^{*}}{\stackrel{\theta}{\longrightarrow}} & M(G) \\
& C_{0}(G),
\end{array}
$$

where $\theta^{*}$ is the Banach space adjoint of $\theta: V \mapsto C_{0}(G)$. Let $\psi$ equal $\chi \circ \theta^{*}$. Then $\psi$ is a linear map from $M(G)$ into $A$. We will now investigate the various properties of $\psi$.

If $f$ is an element of $V$ and $\mu$ is an element of $M(G)$ then:

$$
f(\psi(\mu))=f\left(\chi \circ \theta^{*}(\mu)\right)=\theta^{*}(\mu)(f)=\langle\theta(f), \mu\rangle .
$$

Using this one shows that $\psi$ is continuous from $M(G)$ with the $\sigma(M(G)$, $\left.C_{0}(G)\right)$-topology to $A$ with the $\sigma(A, V)$-topology.

We claim that if $u \in G$ then $\psi\left(\delta_{u}\right)$ is equal to $u$. Since $V$ separates points in $A$ it suffices to show for $f \in V, f\left(\psi\left(\delta_{u}\right)\right)=\left\langle\theta(f), \delta_{u}\right\rangle=\theta(f)(u)=f(u)$. The linearity of $\psi$ implies the above holds for finite linear combinations of point masses of $G$.

Using the above and an argument similar to one given by Rigelhof in [15] one shows that $\psi$ is multiplicative. For completeness sake we outline the argument. The linearity of $\psi, \psi\left(\delta_{u}\right)=u$ for $u \in G$, and an easy calculation shows that $\psi$ is multiplicative on the linear span of the point masses.

For two arbitrary elements, $\mu$ and $\nu$, of $M(G)$ condition (5) allows us to obtain two nets of elements in the linear span of the point masses which converge to $\mu$ and $\nu$. The continuity of $\psi$ and multiplicativity of $\psi$ on pointwise product of the nets shows that $\psi$ is multiplicative on all of $M(G)$. 
Since a Banach space adjoint is always norm decreasing, $\psi$ is norm decreasing and hence $\psi\left(M(G)_{1}\right) \subseteq A_{1}$. Using condition (5), $\psi\left(\delta_{u}\right)=u$ for $u \in G$, the fact $M(G)_{1}$ is $\sigma\left(M(G), C_{0}(G)\right)$-compact, and the weak *-continuity of $\psi$ one shows $\psi\left(M(G)_{1}\right) \supseteq A_{1}$. Thus $\psi$ maps $M(G)_{1}$ onto $A_{1}$.

A straightfoward calculation shows that $\psi$ is a ${ }^{*}$-homomorphism if $\theta$ is a *-homomorphism. Another calculation shows that $\theta$ is a ${ }^{*}$-homomorphism.

Let $N$ equal the kernel of $\psi$. The weak* continuity of $\psi$ implies that $N$ is a $\sigma\left(M(G), C_{0}(G)\right)$-closed (and hence norm closed) ideal in $M(G)$. Since $\psi$ is a *-homomorphism $N$ is a ${ }^{*}$-ideal. Thus $M(G) / N$ is a unital Banach *-algebra. The claims proved above show that $\psi$ induces a norm decreasing *isomorphism, $\Psi$, of $M(G) / N$ onto $A$. To complete the proof of the theorem we need only show that $\Psi$ is isometric. This follows since by Rigelhof [15], the unit ball in $M(G) / N$ is equal to $M(G)_{1} / N$, so $\psi\left(M(G)_{1}\right)=A_{1}$ implies $\Psi\left((M(G) / N)_{1}\right)=A_{1}$. This completes the proof of the theorem.

We will now present a condition that insures $N=\{0\}$. Since $N=\{0\}$ if and only if $\theta(V)$ is dense in $C_{0}(G)$, the most obvious condition is that $\theta(V)$ satisfies the Stone-Weierstrass theorem. In other words:

(7) Suppose that:

1. for each $f \in V$ there exists $f^{\sim} \in V$ such that $f^{\sim}(s)=f(s)^{-}$for each $s \in S$, and furthermore that,

2. for each $f, g \in V$ there exists $h \in V$ such that $f(s) g(s)=h(s)$ for each $s \in S$.

So we have the following theorem.

Theorem 2.2. Let $A$ be a unital Banach *-algebra with isometric involution satisfying the six conditions of Theorem 2.1 and condition (7) above. Then there exists a locally compact group $G$ such that $A$ is isometrically *-isomorphic to $M(G)$.

Next we show that $M(G)$ satisfies the six conditions of Theorem 2.1 and condition (7) above in the following explicit sense:

Theorem 2.3. Let $G$ be a locally compact group. Then $M(G)$ is a unital Banach *-algebra satisfying the six conditions of Theorem 2.1, with $V=\kappa\left(C_{0}(G)\right)$. (Note: if $\omega$ is defined by $\omega(\mu)=\mu(G)$, then $\omega$ satisfies (6)). Futhermore if $\omega$ is any nonzero multiplicative linear functional satisfying condition (6) of Theorem 2.1 and $H=\{s \in S: \omega(s)=1\}$ then the map $\tau: G \mapsto H$ given by $\tau(u)=\omega\left(\delta_{u}\right)^{-1} \delta_{u}$ for all $u \in G$ is a homeomorphic group isomorphism of $G$ onto $H$. Hence $\psi$ is an isometric isomorphism from $M(H)$ onto $M(G)$ where $\psi(\mu)(f)=\int_{H} \omega\left(\delta_{\tau^{-1}(v)}\right)^{-1} f\left(\tau^{-1}(v)\right) d \mu(v)$ for all $f \in C_{0}(G)$ and $\mu \in M(H)$.

Before giving the proof we state the following lemma whose proof is straightforward.

Lemma 2.4. Let $G$ be a locally compact group and $\tilde{S}$ be the following subset of $M(G):\left\{\xi \delta_{u}: \xi \in \mathbf{T}\right.$ and $\left.u \in G\right\}$. Then $\tilde{S}$ with the relative weak ${ }^{*}$ topology is homeomorphically group isomorphic to $\mathbf{T} \times G$.

Proof of Theorem 2.3. We pointed out in $\S 1$ that $\kappa\left(C_{0}(G)\right) \subseteq M(G)^{*}$ is a minimal subspace in $M(G)^{*}$ which is invariant under the induced involution, so conditions (1) and (2) hold with $V=\kappa\left(C_{0}(G)\right)$.

Let $S$ equal $M(G)_{1} \cap M(G)_{U}$ and $\tilde{S}$ equal $\left\{\xi \delta_{u}: \xi \in \mathbf{T}\right.$ and $\left.u \in G\right\}$. 
Claim 1. Then $S$ equals $\tilde{S}$.

Proof. For $\xi \in \mathbf{T}$ and $u \in G,\left(\xi \delta_{u}\right)^{*}=\xi^{-} \delta_{u^{-1}}=\left(\xi \delta_{u}\right)^{-1}$; thus $S \supseteq \tilde{S}$. To show the opposite inclusion let $\mu \in S ;\{e\}$ is a Borel set and hence $|\mu| *\left|\mu^{*}\right|$ measureable so by $[7,19.11]$,

$$
\begin{aligned}
1 & =\delta_{e}(e)=\mu * \mu^{*}(e)=\int_{G} \mu^{*}\left(v^{-1} e\right) d \mu(v)=\int_{G} \mu(v)^{-} d \mu(v) \\
& =\int_{E} \mu(v)^{-} d \mu(v) \quad \text { where } E=\{v \in G: \mu(v) \neq 0\} .
\end{aligned}
$$

Thus $\mu(E) \neq 0, \mu$ restricted to the set $E$ is a discrete measure, and $1=$ $\sum_{v \in E}|\mu(v)|^{2}$. Since the norm of $\mu$ equals one, $1=|\mu|(G) \geq \sum_{v \in G}|\mu(v)| \geq$ $\sum_{v \in E}|\mu(v)|^{2}=1$. Hence $E$ is a singleton, $\operatorname{supp}(\mu)=E$, and $\mu=\left(\xi \delta_{u}\right)$ where $\xi \in \mathbf{T}$ and $u \in G$, and $S$ equals $\tilde{S}$.

In [15] Rigelhof showed that $\tilde{S} \cup\{0\}$ is $\sigma\left(M(G), C_{0}(G)\right)$-closed and the set of exterme points of $M(G)_{1}$ is $\tilde{S}$. Thus by Claim 1 condition (3) holds and by the Krein-Milman theorem condition (5) holds. In [15, Proposition 1] Rigelhof also showed that condition (4) holds for $M(G)$.

Define $\omega$ by $\omega(\mu)=\int_{G} d \mu$. Then $\omega$ is a nonzero multiplicative linear functional and $H=\{v \in S: \omega(v)=1\}=\left\{\delta_{u}: u \in G\right\}$. Lemma 2.4 and Claim 1 imply that condition (6) holds for $\omega$.

We now prove the penultimate sentence of the theorem. Let $\omega$ be any nonzero multiplicative linear functional which is $\sigma\left(M(G), C_{0}(G)\right)$-continuous on $S$.

Let $H=\{s \in S: \omega(s)=1\}$, and define $\tau(u)=\omega\left(\delta_{u}\right)^{-1} \delta_{u}$ for all $u \in G$. Clearly $\tau$ is a group homomorphism. Lemma 2.4 and the $\sigma\left(M(G), C_{0}(G)\right)$ continuity of $\omega$ imply that $\tau$ is a group homeomorphism of $G$ onto $H$.

Finally we must show that $\psi$ has the desired properties. For $f \in C_{0}(G), v \in$ $H, \theta: C_{0}(G) \mapsto C_{0}(H)$ is given by $\theta(f)(v)=\langle f, v\rangle=\omega\left(\delta_{\tau^{-1}(v)}\right)^{-1} f\left(\tau^{-1}(v)\right)$. There is an inverse map $\theta^{-1}: C_{0}(H) \mapsto C_{0}(G)$ which is given by $\theta^{-1}(g)(u)=$ $\omega\left(\delta_{u}\right) \cdot g(\tau(u))$, for $u \in G, g \in C_{0}(H)$. Hence $\theta$ is onto and $\psi$ is one-to-one, since $\psi(\mu)(f)=\langle\theta(f), \mu\rangle$ for $\mu \in M(H), f \in C_{0}(G)$. Recall that in the proof of Theorem 2.1 we had $\psi\left(M(H)_{1}\right)=M(G)_{1}$. Thus $\psi$ is an isometric isomorphism. We have completed the proof of Thereom 2.3.

We now look at the case in which $A$ does not satisfy condition (6) of Theorem 2.1. We have the following theorem.

Theorem 2.5. If $A$ is a unital Banach *-algebra with continuous involution which satisfies conditions (1)-(5) of Theorem 2.1 then there exists a locally compact group $G$ and $\sigma\left(M(G), C_{0}(G)\right)$-closed *-ideal, $N$, of $M(G)$ such that $A$ is isometrically isomorphic to $M(G) / N$. Conversely if $G$ is a locally compact group and $N$ is a $\sigma\left(M(G), C_{0}(G)\right.$ )-closed *-ideal of $M(G)$ then $M(G) / N$ satisfies conditions (1)-(5) of Theorem 2.1.

Proof. If $A$ is a unital Banach *-algebra let $S$ be as in Theorem 2.1. We will take $G$ equal to $S$. The proof that $A$ is isometrically *-isomorphic to $M(G) / N$, where $N$ is the kernel of the adjoint map $\theta: V \mapsto C_{0}(G)$, is almost verbatim the proof given for Theorem 2.1. The only difference in the proof is $\omega$ is not involved in any of the calculations. So we leave the details of the proof for the first half of the theorem to the reader. 
Let $G$ be a locally compact group and $N$ a $\sigma\left(M(G), C_{0}(G)\right)$-closed *-ideal of $M(G)$. It is easily shown that $M(G) / N$ is a unital Banach *-algebra with isometric involution. We will now show that $M(G) / N$ satisfies conditions (1)(5) of Theorem 2.1.

Let $N_{\perp}=\left\{f \in C_{0}(G):\langle f, \mu\rangle=0\right.$ for all $\left.\mu \in N\right\}$ and let $N^{\perp}=\{\omega \in$ $M(G)^{*}: \omega(\mu)=0$ for $\left.\mu \in N\right\}$. Then by [16,4.6-4.9] $N^{\perp}$ is isometrically isomorphic to $(M(G) / N)^{*}$ and $(M(G) / N)$ is isometrically isomorphic to $\left(N_{\perp}\right)^{*}$. Thus by Diximier [2] $\kappa\left(N_{\perp}\right)$ is a minimal subspace of $(M(G) / N)^{*}=N^{\perp}$ and condition (1) is satisfied.

Since the involution on $M(G) / N$ agrees with the involution induced from $N_{\perp}, \kappa\left(N_{\perp}\right)$ is invariant under the induced involution on $(M(G) / N)^{*}$.

In [14] Rigelhof showed the set of extreme points of $(M(G) / N)_{1}$ is the image of the extreme points of $M(G)_{1}$ under $\pi$, where $\pi$ is the natural map from $M(G)$ onto $M(G) / N$. Using the above and that for any Banach *-algebra, $A$, $A_{U} \cap A_{1}$ is contained in the set of extreme points of $A_{1}$ (cf., [3]) one shows that $S_{M(G) / N}$ equals $\pi\left(S_{M(G)}\right)$, where $S$ is defined above.

The above argument also shows that the set of extreme points of $(M(G) / N)_{1}$ is equal to $S_{M(G) / N}$ so by the Krein-Milman theorem the weak * closure of the convex hull of $S_{M(G) / N}$ is equal to $(M(G) / N)_{1}$, hence condition (5) is satisfied by $M(G) / N$.

Now $N$ weak $*$ closed implies that $\left(N_{\perp}\right)^{\perp}$ equals $N$. This implies that the $\sigma\left(M(G) / N, N_{\perp}\right)$-topology is the weak * quotient topology on $M(G) / N$. Hence $S_{M(G) / N} \cup\{0\}$, which is the continuous image of a compact set $S_{M(G)} \cup\{0\}$, is compact in the $\sigma\left(M(G) / N, N_{\perp}\right)$-topology, and thus is $\sigma\left(M(G) / N, N_{\perp}\right)$ closed. The fact that the $\sigma\left(M(G) / N, N_{\perp}\right)$-topology is the weak * quotient topology on $M(G) / N$ also implies that the multiplication on $M(G) / N$ is separately $\sigma\left(M(G) / N, N_{\perp}\right)$-continuous. Thus $M(G) / N$ satisfies conditions (1)-(5) of Theorem 2.1.

\section{The DOUble CENTRALIZER ALGEBRA AND THE STRICT TOPOLOGY}

Centralizer algebras have been studied as mathematical objects cf., [11]. For more complete definitions and proofs of the following see [11] or [3].

Let $A$ be an algebra. A left (respectively, right) centralizer is a linear map $L: A \mapsto A(R: A \mapsto A)$ such that $L(a b)=L(a) b \quad(R(a b)=a R(b))$ for all $a, b \in A$. Denote the set of all left, right centralizers of $A$ by $D_{L}(A), D_{R}(A)$ respectively. We will denote the set of double centralizers of $A$ by $D(A)$ (i.e. the set of all pairs, $(L, R)$, where $L \in D_{L}(A), R \in D_{R}(A)$, and $(L, R)$ satisfies the equation $a L(b)=R(a) b)$. With coordinatewise addition and the multiplication, $\left(L_{1}, R_{1}\right) \cdot\left(L_{2}, R_{2}\right)=\left(L_{1} \circ L_{2}, R_{2} \circ R_{1}\right), D(A)$ is an algebra. For $a \in A$ define two maps, $L_{a}: A \mapsto A$, and $R_{a}: A \mapsto A$, by $L_{a}(b)=a b$, $R_{a}(b)=b a$. The regular homomorphism is the map $\pi: a \mapsto\left(L_{a}, R_{a}\right)$ of $A$ into $D(A)$. If $A$ is a *-algebra and $S: A \mapsto A$ is a linear map define $S^{*}$ by $S^{*}(a)=S\left(a^{*}\right)^{*}$. Then $(L, R)^{*}=\left(R^{*}, L^{*}\right)$ defines an involution on $D(A)$. We will be interested only in elements of $D(A)$ where $L$ and $R$ are norm continuous. There are several conditions that guarantee the continuity of $L$ and $R$. The one most suitable for us is contained in the following theorem.

Theorem 3.1. Let $A$ be a normed *-algebra with isometric involution and a two- 
sided norm one appoximate identity. If $(L, R)$ is a double centralizer then $L$ and $R$ are continuous and hence bounded. Make $D(A)$ into a normed algebra by using the maximum of the operator norms of $L$ and $R$ for $(L, R) \in D(A)$. Then the regular homomorphism is an isometric *-isomorphism of $A$ into the unital normed *algebra $D(A)$, and the involution on $D(A)$ is an isometric involution. Futhermore if $A$ is a Banach *-algebra then the image of $A$ in $D(A)$ is a closed *-ideal and $D(A)$ is a Banach *-algebra.

Definition 3.2. Let $B$ be a normed algebra and $I$ a two-sided ideal of $B$. The strict topology on $B$ is the topology induced by the family of pseudo norms $\|\cdot\|_{b}, \quad b\|\cdot\|$ for $b \in I$, where

$$
\|a\|_{b}=\|a b\|, \quad \text { and }{ }_{b}\|a\|=\|b a\| \quad \text { for all } a \in B \text {. }
$$

Thus a net $\left\{a_{\beta}\right\}_{\beta \in B}$ converges in the strict topology to $a \in A$ if and only if $\lim _{\beta}\left\|a_{\beta} b-a b\right\|=0=\lim _{\beta}\left\|b a_{\beta}-b a\right\|$, for all $b \in I$. We denote the strict topology on $B$ with respect to $I$ by $S(B, I)$. The $S_{L}(B, I)$-topology is defined using only the family of pseudo norms, $b\|\cdot\|$.

The proofs of the following facts and the first half of the proposition are straight forward or see [3]. The proof of the second half of the proposition is exactly the same as the proof of Claim 1, Theorem 2.1.

If $I$ contains a two-sided appoximate identity then $I$ is $S(B, I)$-dense in $B$. Also the multiplication on $B$ is jointly $S(B, I)$-continuous on norm bounded sets. If in addition $B$ is a *algebra with continuous involution and $I$ is a *-ideal then $(*)$ is $S(B, I)$-continuous.

Proposition 3.3. Let $B$ be a normed algebra and $I$ a two-sided ideal which contains a two-sided appoximate identity. Then any nonvanishing continuous multiplicative linear functional, $\omega$, on I has a unique extension, $\omega^{\sim}$, to $B$. This extension is given by $\omega^{\sim}(a)=\omega(a b) / \omega(b)$, where $b$ is any element of $I$ with $\omega(b) \neq 0$. Furthermore $\omega^{\sim}$ is $S(B, I)$-continuous. If in addition $B$ has an isometric involution let $S=B_{U} \cap B_{1}$ and give $S$ the relative $S(B, I)$-topology. Then $S$ is homeomorphically group isomorphic to $\mathbf{T} \times G$, where $G=\left\{u \in S: \omega^{\sim}(u)=1\right\}$ and $G$ has the relative $S(B, I)$-topology.

Let $A$ be a normed *-algebra with isometric involution and a norm one two-sided appoximate identity. If we let $B=D(A)$ and $I=A$, we have the following obvious corollary to Proposition 3.3, but in this case $S$ is also closed in $D(A)$.

Proposition 3.4. Let $A$ be a normed *-algebra with isometric involution and a norm one two-sided appoximate identity. Let $S=D(A)_{U} \cap D(A)_{1}$, and give $S$ the relative $S(D(A), A)$-topology. If $\omega$ is any continuous nonvanishing multiplicative linear functional on $A$, let $\omega^{\sim}$ be the extension of $\omega$ given by Proposition 3.3. Then $S$ is homeomorphically group isomorphic to $\mathbf{T} \times G$, where $G=\left\{u \in S: \omega^{\sim}(u)=1\right\}$ and $G$ has the relative $S(D(A), A)$-topology. Moreover $S$ is a $S(D(A), A)$-closed subset of $D(A)$.

Proof. Except for the last statement the proposition follows directly from Theorem 3.1 and Proposition 3.3. An easy calculation using the $S(D(A), A)$ continuity of multiplication on bounded sets and the $S(D(A), A)$-continuity of the involution shows the $S(D(A), A)$-limit of any net in $S$ is a unitary element. Thus to show $S$ is closed we need only show the $S(D(A), A)$-limit of 
any net in $S$ has norm one. Since any unitary element in a normed *-algebra with isometric involution has norm greater than or equal to one it suffices to prove:

Claim 1. Let $\left\{\left(L_{\beta}, R_{\beta}\right)\right\}_{\beta \in B}$ be a net in $D(A)$ with $S(D(A), A)$-limit equal to $(L, R)$. Then the $\liminf _{\beta}\left\|\left(L_{\beta}, R_{\beta}\right)\right\|$ is greater than or equal to $\|(L, R)\|$.

Proof of claim. Since $\|(L, R)\|=\max \{\|L\|,\|R\|\}$ and $\liminf _{\beta}\left\|\left(L_{\beta}, R_{\beta}\right)\right\| \geq$ $\max \left\{\liminf _{\beta}\left\|L_{\beta}\right\|, \liminf _{\beta}\left\|R_{\beta}\right\|\right\}$, it suffices to prove $\liminf _{\beta}\left\|L_{\beta}\right\| \geq\|L\|$ and $\liminf _{\beta}\left\|R_{\beta}\right\| \geq\|R\|$. But this easily follows from the definition of the strict topology and the operator norm. This completes the proof of the claim and Propositon 3.6.

\section{Characterization of $L^{1}(G)$ IN terms OF THE UNITARY ELEMENTS OF $M(G)$}

Before giving our first characterization of $L^{1}(G)$ we prove the following lemma.

Lemma 4.1. Let $G$ be a locally compact group. Then $L^{1}(G)=\{\mu \in M(G): v \mapsto$ $v * \mu$ and $v \mapsto \mu * v$ are continuous from $S=M(G)_{U} \cap M(G)_{1}$ with the $\sigma\left(M(G), C_{0}(G)\right)$-topology to $M(G)$ with the norm topology\}.

Proof. Let $V$ equal the above set and let $W=\{\mu \in M(G)$ : the maps $u \mapsto$ $\delta_{u} * \mu$ and $u \mapsto \mu * \delta_{u}$ are norm continuous from $G$ into $\left.M(G)\right\}$. It follows, essentially from $\left[8,19.27\right.$ and 20.31] and $[19,3.13]$ that $L^{1}(G)$ equals $W$ and from Lemma 2.4, and Claim 1 of Theorem 2.1 that $V=W$.

Theorem 4.2. Let $A$ be a Banach *-algebra with isometric involution and a norm one approximate identity. Suppose that $D(A)$ satisfies conditions (1)-(7) of Theorem 2.1 and Theorem 2.2, i.e:

(1) There is a minimal subspace $V \subset D(A)^{*}$ such that,

(2) $V$ is invariant under the involution induced on $D(A)^{*}$.

(3) If $S=D(A)_{U} \cap D(A)_{1}$ then $S \cup\{0\}$ is $\sigma(D(A), V)$-closed.

(4) Multiplication in $D(A)$ is separately $\sigma(D(A), V)$-continuous.

(5) The $\sigma(D(A), V)$-closure of $\operatorname{co}[S]$ is equal to $D(A)_{1}$.

(6) There exists a nonzero multiplicative linear functional, $\omega$, which is $\sigma(D(A), V)$-continuous on $S$.

(7) 1. For each $f \in V$ there exists $f^{\sim} \in V$ such that $f^{\sim}(s)=f(s)^{-}$for each $s \in S$, furthermore that,

2. for each $f, g \in V$ there exists $h \in V$ such that $f(s) g(s)=h(s)$ for each $s \in S$.

Futhermore suppose that:

(8) $A=\{b \in D(A): u \mapsto u b$ and $u \mapsto b u$ are continuous from the $\sigma(D(A), V)$-topology on $S$ to the norm topology on $D(A)\}$.

Then $A$ is isometrically *-isomorphic to $L^{1}(G)$.

Proof. By Theorem 2.2 there exists an isometric *-isomophism, $\psi$, from $M(G)$ onto $D(A)$. So it suffices to prove that $\psi\left(L^{1}(G)\right)$ is equal to $A$. We remind the reader of those parts of Theorem 2.1 we will need:

(i) $S$ with the relative $\sigma(D(A), V)$-topology is homeomorphically group isomorphic to $\mathbf{T} \times G$. 
(ii) $\psi$ is continuous from $M(G)$ with the $\sigma\left(M(G), C_{0}(G)\right)$-topology to $D(A)$ with the $\sigma(D(A), V)$-topology.

(iii) For $\zeta \in \mathbf{T}$ and $u \in G$ we have $\psi\left(\zeta \delta_{u}\right)=\zeta u \in S$.

For $a \in A$ a straightfoward calculation using that $\psi$ is an isometry, condition (8), and (i) and (ii) show that $\psi^{-1}(a) \in L^{1}(G)$. Thus $A$ is included in $\psi\left(L^{1}(G)\right)$.

We will now show that $\psi\left(L^{1}(G)\right)$ is included in $A$. Let $f$ be an element of $L^{1}(G)$, and let $v_{1}, v_{2} \in S$. By (i) there exists $\left(\zeta_{1}, u_{1}\right),\left(\zeta_{2}, u_{1}\right) \in \mathbf{T} \times G$ with $\zeta_{i} \cdot u_{i}=v_{i}$, for $i=1,2$. Now $\psi$ an isometry and (ii) implies

$$
\begin{gathered}
\left\|v_{1} \cdot \psi(f)-v_{2} \cdot \psi(f)\right\|=\left\|\zeta_{1} \delta_{u_{1}} * f-\zeta_{2} \delta_{u_{2}} f\right\| \\
\leq\left\|\delta_{u_{1}} * f-\delta_{u_{2}} f\right\|+\left|\zeta_{1}-\zeta_{2}\right|\|f\| .
\end{gathered}
$$

The above equation, (ii), and Lemma 5.1 show that $\psi(f)$ satisfies conditon (8) and hence is an element of $A$.

Since we have already shown that $M(G)$ satisfies conditions (1)-(7) and Lemma 5.1 shows that $L^{1}(G)$ satisfies condition (8), this gives a complete characterization of $L^{1}(G)$.

We will now prove two propositions that will be needed in our second characterization of $L^{1}(G)$.

In Proposition 3.4 we showed that if $S$ equals $D(A)_{U} \cap D(A)_{1}$ and $S$ has the relative $S(D(A), A)$-topology then $S$ is a topological group with a Hausdorff topology. We now make the assumption that $S$ is also a locally compact group so that we can construct an algebra homomorphism from $M(S)$ into $D(A)$. If $u$ is an element of $S$ let $\left(L_{u}, R_{u}\right)$ be its representation as an element of $D(A)$.

Proposition 4.3. Let $A$ be a Banach *-algebra with isometric involution and a norm one approximate unit. Let $S$ equal $D(A)_{U} \cap D(A)_{1}$ and give $S$ the relative $S(D(A), A)$-topology. If $S$ is a locally compact group define $\theta_{L}: M(S) \mapsto D_{L}(A)$ by

$$
\theta_{L}(\mu)(a)=\int_{S} L_{u}(a) d \mu(u),
$$

and define $\theta_{R}: M(S) \mapsto D_{R}(A)$ by

$$
\theta_{R}(\mu)(a)=\int_{S} R_{u}(a) d \mu(u) .
$$

If we let $\theta(\mu)$ equal $\left(\theta_{L}(\mu), \theta_{R}(\mu)\right)$, then $\theta$ is an algebra *-homomophism of $M(S)$ into $D(A)$.

Proof. We first show that the two vector valued integrals defined above make sense. (Note: We can not use the Bochner integral since unless $S$ is second countable the image of $u \mapsto L_{u}(a)$ need not be separable.) We will show for $a \in A$ the map, $u \mapsto L_{u}(a)$, from $S$ into $A$ is Pettis-integrable. Note for a fixed $a \in A, u \mapsto L_{u}(a)$ is continuous from $S$ with the relative $S(D(A), A)$-topology into $A$ with its norm topology. The image is contained in the ball of radius $\|a\|$. Thus if $\omega \in A^{*}$ and $a \in A$ the map $u \mapsto \omega\left(L_{u}(a)\right)$ is absolutely integrable with respect to $\mu$. Hence by Bourbaki [1, Proposition 2.8] for all $a \in A$ the map $u \mapsto L_{u}(a)$ is Pettis-integrable with respect to 
$\mu$, and $\int_{S} L_{u}(a) d \mu(u)$ is an element of $A$. A similar argument shows that $\int_{S} R_{u}(a) d \mu(u)$ is an element of $A$, for all $a \in A$ and $\mu \in M(S)$.

To complete the proof we must show the following: $\theta_{L}(\mu)$ and $\theta_{R}(\mu)$ are left and right centralizers respectively, that $\theta(\mu)$ is a double centralizer, and lastly that $\theta: M(S) \mapsto D(A)$ is linear, multiplicative and preserves the involution. To prove the above, one uses the fact that $A^{*}$ separates points and the following: if $\omega \in A^{*}$ and $a \in A$, denote $\omega(a)$ by $\langle a, \omega\rangle$, then by Bourbaki [1, Proposition 1.1] $\left\langle\int_{S} L_{u}(a) d \mu(u), \omega\right\rangle=\int_{S}\left\langle L_{u}(a), \omega\right\rangle d \mu(u)$, and $\left\langle\int_{S} R_{u}(a) d \mu(u), \omega\right\rangle=\int_{S}\left\langle R_{u}(a), \omega\right\rangle d \mu(u)$.

The arguments used to prove each of the items listed above are similar. For example to show that $\theta_{R}(\mu)$ is a right centalizer one shows $\left\langle\theta_{R}(\mu)(a b), \omega\right\rangle$ equals $\left\langle a \cdot \theta_{R}(\mu)(b), \omega\right\rangle$. To give the flavor of all the arguments and since the argument is slightly more complicated we will show that $\theta: M(S) \mapsto D(A)$ is multiplicative and then outline the argument showing that $\theta$ is a ${ }^{*}$-homomorphism.

Let $\mu$ and $\nu$ be elements of $M(S)$. Note that

$$
\begin{aligned}
\theta(\mu * \nu) & =\left(\theta_{L}(\mu * \nu), \theta_{R}(\mu * \nu)\right) \quad \text { and } \\
\theta(\mu) \circ \theta(\nu) & =\left(\theta_{L}(\mu) \circ \theta_{L}(\nu), \theta_{R}(\nu) \circ \theta_{R}(\mu)\right) .
\end{aligned}
$$

Thus to prove $\theta(\mu * \nu)=\theta(\mu) \circ \theta(\nu)$ it suffices to show $\theta_{L}(\mu) \circ \theta_{L}(\nu)=\theta_{L}(\mu * \nu)$ and $\theta_{R}(\nu) \circ \theta_{R}(\mu)=\theta_{R}(\mu * \nu)$. We will show the second equality. Let $a \in A$ and $\omega \in A^{*}$; then we have

$$
\begin{aligned}
\left\langle\theta_{R}(\nu) \circ \theta_{R}(\mu), \omega\right\rangle & =\int_{S}\left\langle R_{u}(a), \theta_{R}(\nu)^{*}(\omega)\right\rangle d \mu(u) \\
& =\int_{S} \int_{S}\left\langle R_{u} \cdot R_{v}(a), \omega\right\rangle d \nu(v) d \mu(u) \\
& =\int_{S} \int_{S}\left\langle R_{u v}(a), \omega\right\rangle d \nu(v) d \mu(u) .
\end{aligned}
$$

As noted above the function $u \mapsto\left\langle R_{u}(a), \omega\right\rangle$ is a bounded continuous function on $S$ and thus absolutely integrable with respect to $|\mu| *|\nu|$. Hence by $[8,19.10]$ we have

$$
\left\langle\theta_{R}(\nu) \circ \theta_{R}(\mu), \omega\right\rangle=\int_{S}\left\langle R_{u}(a), \omega\right\rangle d \mu * \nu(u)=\left\langle\theta_{R}(\mu * \nu)(a), \omega\right\rangle .
$$

We will now outline the argument that shows $\theta$ is a ${ }^{*}$-homomorphism. A calculation using the definition of the involution induced on $D(A)$ by $A$ shows $\theta$ is a ${ }^{*}$-homomorphism if $\theta_{L}\left(\mu^{*}\right)=\theta_{R}(\mu)^{*}$ and $\theta_{R}\left(\mu^{*}\right)=\theta_{L}(\mu)^{*}$. For $u \in S,\left(L_{u^{-1}}, R_{u^{-1}}\right)=u^{-1}=u^{*}=\left(L_{u}, R_{u}\right)^{*}=\left(R_{u}{ }^{*}, L_{u}{ }^{*}\right)$ implies that $R_{u}{ }^{*}=$ $L_{u^{-1}}$. Using this fact along with the definitions of the involutions induced by $A$ on $A^{*}$ (cf. §1) and on $D(A)$ (cf. §3) one shows $\left\langle\theta_{R}(\mu)^{*}(a), \omega\right\rangle=\left\langle\theta_{L}\left(\mu^{*}\right), \omega\right\rangle$, for $\omega \in A^{*}$ and $a \in A$. This completes the proof of the proposition.

Suppose in the above situation there is also a nonzero multiplicative linear functional, $\omega$, defined on $A$ then by Proposition 3.3, $S$ is homeomorphically group isomorphic to $\mathbf{T} \times G$, where $G=\{u \in S: \omega(u)=1\}$. By Lemma A.1, $G$ is locally compact. We could then define $\theta$ on $G$ and using the same proof as above have the following proposition. 
Proposition 4.4. Let $A$ be a Banach *-algebra with isometric involution and a norm one approximate unit. Let $S$ equal $D(A)_{U} \cap D(A)_{1}$ and give $S$ the relative $S(D(A), A)$-topology. Suppose there exists a nonvanishing multiplicative linear functional, $\omega$, on $A$ and $S$ is a locally compact. Let $G=\{u \in S: \omega(u)=1\}$, and define $\theta_{L}: M(G) \mapsto D_{L}(A)$ by

$$
\theta_{L}(\mu)(a)=\int_{G} L_{u}(a) d \mu(u),
$$

and define $\theta_{R}: M(G) \mapsto D_{R}(A)$ by

$$
\theta_{R}(\mu)(a)=\int_{G} R_{u}(a) d \mu(u) .
$$

If we let $\theta(\mu)$ equal $\left(\theta_{L}(\mu), \theta_{R}(\mu)\right)$, then $\theta$ is an algebra *-homomophism of $M(G)$ into $D(A)$.

We are now ready to give our second characterization of $L^{1}(G)$. As we noted above some of the conditions are stronger than we would like. (For example conditions (1), (5), (6).)

Proposition 4.5. Let $A$ be a Banach *-algebra with isometric involution and a norm one approximate unit. Let $S$ equal $D(A)_{U} \cap D(A)_{1}$. Suppose:

(1) In the relative $S(D(A), A)$-topology $S$ is locally compact.

(2) There exists a nonvanishing norm continuous multiplicative linear functional, $\omega$ on $A$. Let $G$ equal $\{u \in S: \omega(u)=1\}$

(3) The convex hull of $S$ is dense in $D(A)_{1}$ in the $S(D(A), A)$-topology.

(4) $D(A)^{*}$ contains a minimal subspace $V$, on norm bounded sets the $S(D(A), A)$-topology is at least as fine as the $\sigma(D(A), V)$-topology, and on $S$ the two topologies agree.

(5) If $\theta$ is defined as in Proposition 4.4 then $\theta$ is continuous from $M(G)$, with the $\sigma\left(M(G), C_{0}(G)\right)$-topology, into $D(A)$ with the $S(D(A), A)$ topology.

(6) If $\theta$ is defined as in Proposition 4.4 then the kernel of $\theta$ is equal to $\{0\}$.

(7) $A=\{b \in D(A): u \mapsto u b$ and $u \mapsto b u$ are continuous from the $\sigma(D(A), V)$-topology on $S$ to the norm topology on $D(A)\}$.

Then $A$ is isometrically *-isomorphic to $L^{1}(G)$.

Proof. If $G$ equals $\{u \in S: \omega(u)=1\}$; then by Proposition $4.4 G$ is a locally compact group and $\theta: M(G) \mapsto D(A)$ as defined in Proposition 4.4 is a ${ }^{*}$-homomorphism. Since $\|u\|=\left\|L_{u}\right\|=1$, Bourbaki [1, Proposition 1.2.6] implies

$$
\begin{aligned}
\left\|\theta_{L}(\mu)(a)\right\| & =\left\|\int_{S} L_{u}(a) d \mu(u)\right\| \leq \int_{S}\left\|L_{u}(a)\right\| d|\mu|(u) \\
& \leq \int_{S}\left\|L_{u}\right\|\|(a)\| d|\mu|(u)=\|a\|\|\mu\| .
\end{aligned}
$$

Similarly $\left\|\theta_{R}(\mu)(a)\right\|$ is less than or equal to $\|a\|\|\mu\|$. Thus $\theta$ is norm decreasing.

Next we will show that $\theta$ is onto. Denote the convex hull of $S$ by $D$ and the convex hull of $M(G)_{U} \cap M(G)_{1}$ by $D^{\sim}$. Note for $u \in G$ and $\zeta \in \mathbf{T}, \theta\left(\zeta \delta_{u}\right)=\zeta\left(L_{u}, R_{u}\right)$; thus $\theta$ sends $D^{\sim}$ onto $D$. Since the $S(D(A), A)$ - 
topology is finer than the $\sigma(D(A), V)$-topology on norm bounded sets, condition (2) implies that $D$ is $\sigma(D(A), V)$-dense in $D(A)_{1}$. A quick calculation using the compactness of $M(G)_{1}$ in the $\sigma\left(M(G), C_{0}(G)\right)$-topology, condition (4), and the above comments show that $\theta\left(M(G)_{1}\right)=D(A)_{1}$ and hence that $\theta$ is onto.

The observation $\theta\left(M(G)_{1}\right)$ equals $D(A)_{1}$, and condition (6) implies that $\theta$ is an isometry. We have now shown that $\theta$ is an isometric ${ }^{*}$-isomorphism of $M(G)$ onto $D(A)$.

We must now show that $\theta\left(L^{1}(G)\right)$ equals $A$. By condition (4) and Proposition 3.4, $S$ with relative $\sigma(D(A), V)$-topology is homeomorphically group isomorphic to $\mathbf{T} \times G$. The argument used in Theorem 4.2 now shows that $\theta\left(L^{1}(G)\right)$ equals $A$.

Before showing that $L^{1}(G)$ satisfies the hypotheses of Theorem 4.5 we give a few preparatory remarks.

Definition 4.6. Let $A$ be a normed linear space, and let $V$ be a subset of $A^{*}$. Let $d$ be the pseudo-metric on $A$ defined by $d(a, b)=\|\| a\|-\| b \| \mid$, for $a, b \in$ $A$. Then the $\sigma v(A, V)$-topology is the coarsest topology that contains both the $\sigma(A, V)$-topology and the topology defined by $d$.

In [13,4.2] McKennon shows: Let $G$ be a locally compact group and $\left\{\mu_{\beta}\right\}_{\beta \in B}$ be a net in $M(G)$ with the $\sigma v\left(M(G), C_{0}(G)\right)$-limit of $\left\{\mu_{\beta}\right\}_{\beta \in B}$ equal to $\mu$. Then $\left\{\mu_{\beta}\right\}_{\beta \in B}$ also converges to $\mu$ in the $S_{L}\left(M(G), L^{1}(G)\right)$-topology.

Since $(*)$ on $M(G)$ is both isometric and $\sigma\left(M(G), C_{0}(G)\right)$-continuous, a moments thought shows that $(*)$ is $\sigma v\left(M(G), C_{0}(G)\right)$-continuous. The last fact, McKennon's result, and an easy calculation show the following:

Proposition 4.7. Let $G$ be a locally compact group and $\left\{\mu_{\beta}\right\}_{\beta \in B}$ be a net in $M(G)$ with the $\sigma v\left(M(G), C_{0}(G)\right)$-limit of $\left\{\mu_{\beta}\right\}_{\beta \in B}$ equal to $\mu$. Then $\left\{\mu_{\beta}\right\}_{\beta \in B}$ also converges to $\mu$ in the $S\left(M(G), L^{1}(G)\right)$-topology.

McKennon also shows in [13, Lemma 4.15] that on norm bounded sets the $S\left(M(G), L^{1}(G)\right)$-topology is at least as fine as the $\sigma\left(M(G), C_{0}(G)\right)$-topology. Thus Proposition 4.7 implies that on norm bounded sets the $S\left(M(G), L^{1}(G)\right)$ topology and the $\sigma\left(M(G), C_{0}(G)\right)$-topology agree. We are now ready to prove:

Theorem 4.8. Let $G$ be a locally compact group; then with $V$ equal $C_{0}(G)$, $L^{1}(G)$ satisfies the hypotheses of Theorem 4.5.

Proof. As stated in $\S 1, D\left(L^{1}(G)\right)$ is $M(G)$ and $\kappa\left(C_{0}(G)\right)$ is a minimal subspace of $M(G)^{*}$. The paragraph above shows that on $S$ the $S\left(M(G), L^{1}(G)\right.$ )topology and the $\sigma\left(M(G), C_{0}(G)\right)$-topology agree. Lemma 2.4 then implies $S$ with the $S\left(M(G), L^{1}(G)\right)$-topology is homeomorphically group isomorphic to $\mathbf{T} \times G$ and thus is locally compact. As stated above McKennon showed on norm bounded sets the $S\left(M(G), L^{1}(G)\right)$-topology is at least as fine as the $\sigma\left(M(G), C_{0}(G)\right)$-topology. The map $\mu \mapsto \int_{G} d \mu$ is a multiplicative linear functional which is not identically zero on $L^{1}(G)$. We have shown that $L^{1}(G)$ satisfies conditions (1), (2), and (4).

We showed in Theorem 2.3 the $\operatorname{co}[S]$ is $\sigma\left(M(G), C_{0}(G)\right)$-dense in $M(G)_{1}$. Using this, one shows that the $\operatorname{co}[S]$ is also $\sigma v\left(M(G), C_{0}(G)\right)$-dense in $M(G)_{1}$ and thus by Proposition 4.7 the convex hull of $S, \operatorname{co}[S]$ is $S\left(M(G), L^{1}(G)\right.$ )dense in $M(G)_{1}$. 
Before showing that $L^{1}(G)$ satisfies conditions (5) and (6) we give an explicit formula for the map $\theta$ defined in Proposition 4.3. If $\omega$ is the functional defined above let $H=\left\{\zeta \delta_{u}: \omega\left(\zeta \delta_{u}\right)=1\right\}=\left\{\delta_{u}: u \in G\right\}$ and give $H$ the relative $S\left(M(G), L^{1}(G)\right)$-topology. By above arguements the map $\tau: H \mapsto G$, given by $\tau\left(\delta_{u}\right)=u$ defines a homeomorphic group isomorphism from $H$ onto $G$. For $\mu \in M(H)$ we define $\mu^{\sim} \in M(G)$ by

$$
\begin{aligned}
\left\langle\mu^{\sim}, f\right\rangle & =\int_{G} f(u) d \mu^{\sim}(u)=\int_{G} f(u) d \mu\left(\tau^{-1}(u)\right) \\
& =\int_{H} f\left(\tau\left(\delta_{u}\right)\right) d \mu\left(\delta_{u}\right), \quad \text { for } f \in C_{0}(G) .
\end{aligned}
$$

By [10, p. 188], $\mu \mapsto \mu^{\sim}$ is an isometric isomorphism of $M(H)$ onto $M(G)$.

Claim 1. If $\theta$ is the map defined in Proposition 4.3 then $\theta$ is equal to the map $\mu \mapsto \mu^{\sim}$.

Proof. Let $g \in L^{1}(G), f \in C_{0}(G)$, and $\mu \in M(H)$ be given; then we have

$$
\begin{aligned}
\left\langle\theta_{L}(\mu)(g), f\right\rangle & =\int_{H}\left\langle L_{\delta_{u}}(g), f\right\rangle d \mu\left(\delta_{u}\right)=\int_{H}\left\langle\delta_{u} * g, f\right\rangle d \mu\left(\delta_{u}\right) \\
& =\int_{H}\left[\int_{G} g\left(u^{-1} v\right) f(v) d \lambda(v)\right] d \mu\left(\delta_{u}\right) \\
& =\int_{G}\left[\int_{H} g\left(\tau\left(\delta_{u^{-1}}\right) d \mu\left(\delta_{u}\right)\right] f(v) d \lambda(v)\right. \\
& =\int_{G}\left[\int_{G} g\left(u^{-1} v\right) d \mu^{\sim}(u)\right] f(v) d \lambda(v) \\
& =\int_{G}\left[\mu^{\sim} * g(v)\right] f(v) d \lambda(v)=\left\langle\left(\mu^{\sim} * g\right), f\right\rangle .
\end{aligned}
$$

Thus $\theta_{L}(\mu)$ equals $L_{\mu^{\sim}}$. A similar argument show that $\theta_{R}(\mu)$ equals $R_{\mu} \sim$. Hence $\theta$ is equal to the map $\mu \mapsto \mu^{\sim}$ as claimed.

We now return to showing that conditions (5) and (6) are satisfied. Since $\mu \mapsto$ $\mu^{\sim}$ is an isomorphism, condition (6) is clearly satisfied. In Claim 1 we showed that $\theta$ was the Banach adjoint of the map $\tau^{*}: C_{0}(G) \mapsto C_{0}(H)$ that $\tau: H \mapsto$ $G$ induces. Thus $\theta$ is continuous from $M(H)$ with the $\sigma\left(M(H), C_{0}(H)\right)$ topology to $M(G)$ with the $\sigma\left(M(G), C_{0}(G)\right)$-topology.

In $[10$, p. 188] B. Johnson showed that any isometric *-isomorphism, $\varphi$ : $M\left(G_{1}\right) \mapsto M\left(G_{2}\right)$ has the form $\varphi(\mu)(F)=\int_{\tau(F)} \chi(u) d \mu(u)$, for any $\mu \in M\left(G_{1}\right)$ and $F$ any compact set in $G_{2}$, where $\chi$ is a continuous character on $G_{1}$, and $\tau: G_{2} \mapsto G_{1}$ is a topological isomorphism. Thus in particluar $\varphi$ maps $L^{1}\left(G_{1}\right)$ into $L^{1}\left(G_{2}\right)$. In Theorem 4.2 we began with the data $(\omega, V, G)$ and constructed an isometric *-isomorphism $\psi: M(G) \mapsto D(A)$ such that $\psi\left(L^{1}(G)\right)=\pi(A)$, where $\pi: A \mapsto D(A)$ is the natural embedding. By applying Theorem 4.5 and Johnson's result we can give an explicit geometric formula for the map $\psi$ of Theorem 4.2 .

Theorem 4.9. Suppose that a Banach *-algebra, $A$, satisfies the hypotheses of Theorem 4.2, with data $(\omega, V, G)$. Let $\psi: M(G) \mapsto D(A)$ be the isometric 
*-isomorphism defined there. If we write $\psi(\mu) \in D(A)$ as $\left(\psi_{L}(\mu), \psi_{R}(\mu)\right)$ and denote $u$ by $\left(L_{u}, R_{u}\right)$ for $u \in G$, then $\psi$ has the form

$$
\begin{aligned}
& \psi_{L}(\mu)(a)=\int_{G} L_{u}(a) d \mu(u) \quad \text { for all } a \in A, \quad \text { and } \\
& \psi_{R}(\mu)(a)=\int_{G} R_{u}(a) d \mu(u) \quad \text { for all } a \in A .
\end{aligned}
$$

Proof. Let $\omega_{0}$ be the multiplicative linear functional on $D(A)$ defined by $\omega_{0}(T)=\int_{G} \psi^{-1}(T)$. Then $\omega_{0}$ is the image of the linear functional $\mu \mapsto \int_{G} d \mu$ under $\left(\psi^{-1}\right)^{*}$. Since $\psi\left(L^{1}(G)\right)=\pi(A)$, we have $\omega_{0}$ is nonzero. We remind the reader we defined $\psi$ in the proof of Theorem 2.1 and showed $\psi\left(\zeta \cdot \delta_{u}\right)=\zeta u$ for $\zeta \in \mathbf{T}$ and $u \in G$. Hence $\left\{u \in S: \omega_{0}(u)=1\right\}$ equals $\{u \in S: \omega(u)=$ $1\}=G$. Define $V_{0}=\left(\psi^{-1}\right)^{*}\left(\kappa\left(C_{0}(G)\right)\right) \subset D(A)^{*}$. Then using Theorem 4.8 one easily sees the data $\left(\omega_{0}, V_{0}, G\right)$ satisfies Theorem 4.5 . Hence by Theorem 4.5 the map $\theta: M(G) \mapsto D(A)$, where $\theta(\mu)$ equals $\left(\theta_{L}(\mu), \theta_{R}(\mu)\right)$, with

$$
\theta_{L}(\mu)(a)=\int_{G} L_{u}(a) d \mu(u) \text { and } \theta_{R}(\mu)(a)=\int_{G} R_{u}(a) d \mu(u),
$$

is an isometric *-isomorphism of $M(G)$ onto $D(A)$. Note that both $\psi\left(\delta_{u}\right)$ and $\theta\left(\delta_{u}\right)$ are equal to $u$. Thus $\theta^{-1} \circ \psi$ is an isometric isomorphism of $M(G)$ onto $M(G)$ with $\theta^{-1} \circ \psi\left(\delta_{u}\right)=\delta_{u}$, for $u \in G$. Johnson's result shows any isometric isomorphism is determined by its image of the point masses. So $\theta^{-1} \circ \psi$ is the identity isomorphism and $\psi$ is equal to $\theta$ as claimed.

\section{APPENDIX}

Lemma A.1. If $H$ and $G$ are topological spaces with $H \times G$ a locally compact Hausdorff space then $G$ and $H$ are locally compact Hausdorff topological spaces. Proof. By symmetry it suffices to show that $G$ is locally compact and Hausdorff. By Kelly [12, p. 147] $G$ is locally compact.

We next show that $G$ is Hausdorff. Let $e$ be a fixed element of $H$ and let $u_{1}$ and $u_{2}$ be arbitrary elements of $G$. Since $H \times G$ is Hausdorff there exists basic open sets $V_{1} \times U_{1}$ and $V_{2} \times U_{2}$ of $\left(e, u_{1}\right)$ and $\left(e, u_{2}\right)$ respectively with $\left(V_{1} \times U_{1}\right) \cap\left(V_{2} \times U_{2}\right)=\varnothing$, and $U_{1}, U_{2}$ open neighborhoods of $u_{1}$ and $u_{2}$ respectively. Now $e$ an element of both $V_{1}$ and $V_{2}$ implies $U_{1} \cap U_{2}=\varnothing$.

Lemma A.2. Let $G$ be a locally compact group. Let $V$ be a neighborhood of the identity, $e$. Then there exists a symmetric neighborhood, $U$, of e with $U^{-}$ compact and such that $U \cdot(G \backslash V) \cap U=(G \backslash V) \cdot U \cap U$ is equal to the empty set. Proof. Since multiplication is continuous there exists two neighborhoods, $U_{1}$ and $U_{2}$, of $e$ with $U_{1} \cdot U_{2}$ contained in $V$. We may assume that $U_{1}=U_{1}^{-1}$, that $U_{2}=U_{2}^{-1}$, and that $U_{1}$ and $U_{2}$ have compact closures. Let $U$ equal $U_{1} \cap U_{2}$. Then $U$ is a symmetric neighborhood of $e$ with compact closure. This $U$ satisfies the conclusion of the lemma, since if $U \cdot(G \backslash V) \cap U$ is not empty there exists $v \in(G \backslash V)$, and $u_{1}, u_{2} \in U$ with $v u_{1}=u_{2}$. But then $v=u_{2} u_{1}^{-1}$ which since $U \cdot U^{-1} \subset\left(U_{1} \cdot U_{2}\right) \subset V$ contradicts $v \in(G \backslash V)$. A similar argument shows that $(G \backslash V) \cdot U \cap U$ is equal to the empty set.

In [15] R. Rigelhof uses a map similar to the map $m$ used in the proof of Theorem 2.1. He claims that his map, again called $m$, is an open mapping. An easy calculation shows the following. 
Claim. Let $A$ be a Banach algebra. Let $S$ be a subset of $A$ such that $S$ is closed under multiplication and $\lambda s \in S$ for all $\lambda \in \mathbf{T}, s \in S$. Assume that

(1) There is a Banach space $E$ with $E^{*}=A$.

(2) There is a nonzero norm continuous multiplicative linear function $\omega$ on $A$.

Define $G=\{u \in S: \omega(u)=1\}$. Give both $S$ and $G$ the relative $\sigma(A, E)$ topology. Define $m: \mathbf{T} \times G \mapsto S$ by $m(\lambda, g)=\lambda g$ for all $\lambda \in \mathbf{T}, g \in G$. Then $m$ is a homeomorphism if and only if $\omega$ is weak* continuous on $S$.

Rigelhof uses the claim that $m$ is an open mapping in the proof of his characterization of $M(G)$. By Lemma 2.4 the multplicative linear functionals which are induced from group characters are $\sigma\left(M(G), C_{0}(G)\right)$-continuous on $S=\left\{\xi \delta_{u}: \xi \in \mathbf{T}, u \in G\right\}$. Since for any group there is the trivial character, there do exist multiplicative linear functionals which are $\sigma\left(M(G), C_{0}(G)\right)$ continuous on $S$. We are unable to see how Rigelhof's additional hypotheses imply that any multiplicative linear functional is $\sigma\left(M(G), C_{0}(G)\right)$-continuous on $S$.

Since any multiplicative linear functional that does not vanish on $L^{1}(G)$ is induced from a group character, to find a counterexample we would need a multiplicative linear functional which vanishes on $L^{1}(G)$ but is not weak * continuous on $S$. It has been shown that there exist multiplicative linear functionals on $M(G)$ which do not vanish on $L^{1}(G)$; see [7]. But to the author's knowledge their values on $S$ are unknown. The author is unable to provide a counterexample to Rigelhof's full hypothesis.

Let $\mathbf{Z}$ be the integers. We will give an example in which there exists a Banach space, $E$, with $E^{*}=M(\mathbf{Z})$, and a multiplicative linear functional, $\omega$, such that $\omega$ is not $\sigma(M(\mathbf{Z}), E)$-continuous on $S$.

Let $E$ equal $c(\mathbf{Z})$ where $c(\mathbf{Z})$ is the algebra of all functions $f: \mathbf{Z} \mapsto \mathbf{C}$ such that the limits as $n$ goes to either plus or minus infinity exists and both limits are equal. Then it is well known that $l^{1}(\mathbf{Z})$ is equal to $M(\mathbf{Z})$ and that $c(\mathbf{Z})^{*}=l^{1}(\mathbf{Z})$. One checks that if $\left\{\delta_{n}\right\}_{n \in \mathbf{Z}^{+}}$is the sequence of positive point masses then $\left\{\delta_{n}\right\}_{n \in \mathbf{Z}^{+}}$converges in the $\sigma\left(l^{1}(\mathbf{Z}), c(\mathbf{Z})\right.$ )-topology to $\delta_{0}$. Let $\theta$ be an element of $(0,2 \pi)$ and let $\zeta_{\theta}$ be the group character defined by $\zeta_{\theta}(n)=e^{i n \theta}$ for $n \in \mathbf{Z}$. Denote the multiplicative linear functional induced by $\zeta_{\theta}$ also by $\zeta_{\theta}$. Then clearly $\zeta_{\theta}\left(\delta_{n}\right)=e^{i n \theta}$ does not converge to $\zeta_{\theta}\left(\delta_{0}\right)=1$ as $n$ goes to infinity. Thus $\zeta_{\theta}$ is not $\sigma\left(l^{1}(\mathbf{Z}), c(\mathbf{Z})\right)$-continuous on $S$.

\section{REFERENCES}

1. N. Bourbaki, Integration, Chapter VI, Hermann, Paris, 1959.

2. J. Dixmier, Sur un théorème de Banach, Duke Math. J. 15 (1948), 1057-1071.

3. R. S. Doran and T. W. Palmer, General theory of *-algebras, Cambridge Univ. Press (to appear).

4. R. Ellis, Locally compact transformation groups, Duke Math. J. 24 (1957), 119-125.

5. I. Gelfand and M. Naimark, On the embedding of normed rings into the ring of operators in Hilbert space, Mat. USSR-Sb. 12 (1943), 197-213.

6. F. P. Greenleaf, Characterization of group algebras in terms of their translation operators, Pacific J. Math. 18 (1965), 243-276.

7. E. Hewitt and S. Kakutani, Some multiplicative functionals on $M(G)$, Ann. of Math. 79 (1979), 489-505. 
8. E. Hewitt and K. Ross, Abstract harmonic analysis, Vol. 2, Springer-Verlag, Berlin, 1979.

9. __ Abstract harmonic analysis, Vol. 2, Springer-Verlag, Berlin, 1970.

10. B. Johnson, Isometric isomorphisms of measure algebras, Proc. Amer. Math. Soc. 15 (1964), 186-188.

11. $\ldots$ An introduction to the theory of centralizers, Proc. London Math. Soc. 14 (1964), 299-320.

12. J. L. Kelley, General topology, Springer-Verlag, New York, 1961.

13. K. McKennon, Multipliers and positive functionals, Mem. Amer. Math. Soc. 71 (1971).

14. M. A. Rieffel, A characterization of commutative group algebras and measure algebras, Trans. Amer. Math. Soc. 116 (1965), 32-65.

15. R. Rigelhof, A characterization of $M(G)$, Trans. Amer. Math. Soc. 136 (1969), 373-379.

16. W. Rudin, Functional analysis, McGraw-Hill, New York, 1973.

17. S. Sakai, A characterization of $W^{*}$-algebras, Pacific J. Math. 6 (1956), 763-773.

18. _,$C *$-algebras and $W^{*}$-algebras, Springer-Verlag, New York, 1971.

19. G. L. G. Sleijpen, Locally compact semigroups and continuous translations of measures, Proc. London Math. Soc. 37 (1978), 75-97.

20. J. G. Wendel, Left centralizers and isomorphisms of group algebras, Pacific J. Math. 2 (1952), 251-261.

Department of Mathematics, St. Louis University, St. Louis, Missouri 63103 